\title{
Sexual dimorphism in autoimmunity
}

\author{
Kira Rubtsova, ${ }^{1,2}$ Philippa Marrack, , 1,2,3,4 and Anatoly V. Rubtsov ${ }^{1,2}$ \\ ${ }^{1}$ Howard Hughes Medical Institute, Denver, Colorado, USA. ${ }^{2}$ Department of Biomedical Science, National Jewish Health and Department of Immunology and Microbiology, University of Colorado \\ Health Sciences Center, Denver, Colorado, USA. ${ }^{3}$ Department of Medicine, University of Colorado School of Medicine, Aurora, Colorado, USA. ${ }^{4}$ Department of Biochemistry and Molecular Cenetics, \\ University of Colorado, Health Sciences Center, Aurora, Colorado, USA.
}

\begin{abstract}
Autoimmune diseases occur when the immune system attacks and destroys the organs and tissues of its own host. Autoimmunity is the third most common type of disease in the United States. Because there is no cure for autoimmunity, it is extremely important to study the mechanisms that trigger these diseases. Most autoimmune diseases predominantly affect females, indicating a strong sex bias. Various factors, including sex hormones, the presence or absence of a second $X$ chromosome, and sex-specific gut microbiota can influence gene expression in a sex-specific way. These changes in gene expression may, in turn, lead to susceptibility or protection from autoimmunity, creating a sex bias for autoimmune diseases. In this Review we discuss recent findings in the field of sex-dependent regulation of gene expression and autoimmunity.
\end{abstract}

\section{Introduction}

Differences between female and male immune and autoimmune responses have been well documented (1). In general, the frequency and severity of various infectious diseases are higher in males than in females, suggesting that females have stronger immune responses $(2,3)$. The flip side of this phenomenon is that females are more likely to develop autoimmune diseases. In fact, $80 \%$ of autoimmune patients are women. Diseases such as systemic lupus erythematosus (SLE), Grave's disease, Hashimoto's thyroiditis, and Sjögren's syndrome have the greatest female biases, occurring between seven and ten times more frequently in females than in males. MS, RA, and scleroderma also bias toward females, exhibiting, a 2:1-3:1 female/male ratio (2). It must be noted that there are a few autoimmune syndromes that are sex neutral in humans (but not in mice), including type 1 diabetes (T1D) in children under 15 years of age (4); also, some autoimmune disorders such as ankylosing spondylitis and T1D in 15- to 34 -year-old adults occur more often in men (5-8). Such diseases will not be considered in this Review, which will instead focus on autoimmune diseases that occur more frequently in females.

Fundamentally there are three factors that could govern the differences between female and male immune systems. These are the sex hormones themselves, the presence in the host of two $\mathrm{X}$ chromosomes versus one $\mathrm{X}$ and one $\mathrm{Y}$ chromosome, and environmental and societal differences, for example in diet (9). The last of these is unlikely to affect mice, and since female and male mice manifest, on the whole, the same gender biases in autoimmunity as humans do, here we concentrate on the potential roles of sex hormones and X and Y chromosome content. Downstream, these two phenomena may govern directly or indirectly a number of factors, such as estrogen-induced and $\mathrm{X}$-linked genes and gut microflora that contribute to or prevent disease (Figure 1).

Conflict of interest: The authors have declared that no conflict of interest exists. Reference information: J Clin Invest. 2015;125(6):2187-2193. doi:10.1172/JCI78082.

\section{Sex hormones affect the incidence of autoimmunity}

Evidence for the influence of sex hormones on the development of autoimmune diseases includes observed changes in disease severity during pregnancy. The issue of pregnancy is confused by the fact that the condition has opposite effects in humans on some autoimmune diseases, for example the contrasting effects on RA and SLE (reviewed recently by Hughes and Choubey, ref. 10). The classic study that in 1938 reported the amelioration of RA during pregnancy (11) illustrated the protective effects of pregnancy. Later, diseases such as MS were added to the group in which symptoms are reduced during pregnancy (12). On the other hand, many studies have indicated that pregnancy and estrogens (as opposed to progesterone) make the symptoms of SLE and related diseases more severe (reviewed by Jara et al., ref. 13). The different effects of pregnancy on different female-biased autoimmune diseases suggest that the role of sex in these diseases may not always be the same. Alternatively, the fact of being female may initiate disease via the same route in all cases, but pregnancy and sex hormones might differ in their abilities to exacerbate disease in some instances.

Estrogens clearly also affect autoimmunity in experimental animals. Increased estrogen and/or prolactin accelerate-lupus-like disease in NZB/NZW F1 mice $(14,15)$; these effects are dependent upon estrogen receptor $\alpha(E R \alpha)(16)$. However, there is some disagreement on this point (17), and clearer results have been obtained from studies examining the protective effects of androgens.

In human studies, treatment with testosterone had some benefit to men with MS; however, in females with SLE, testosterone administration did not result in significant improvement (18, 19). Testosterone frequently appears to be protective in strains of mice that are autoimmune prone. For example, NZB/NZW F1 males develop disease later than females of the same strain, while disease onset in castrated males approaches that of females $(14,20)$. Similar results have been found for the development of T1D in NOD mice (21) and the onset of arthritis in SKG male mice injected with zymosan, a procedure that quickly induces disease in female but not normal male mice of this strain (22). 


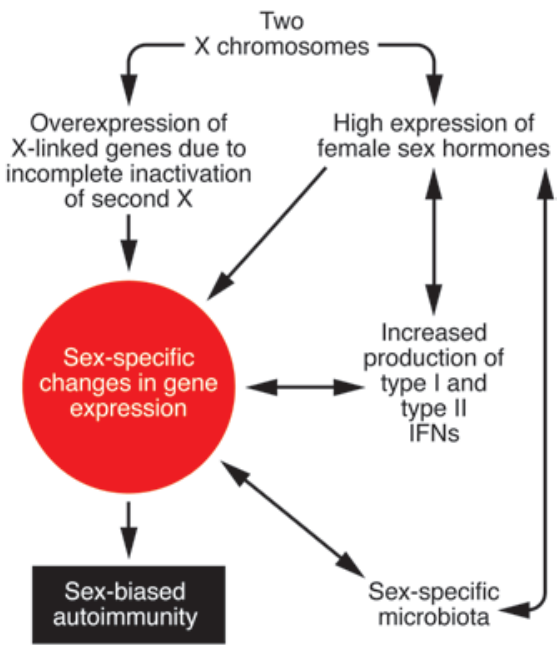

Figure 1. Sex-specific factors that lead to sex bias in autoimmunity. Sexrelated factors, including sex hormones, the presence of two $X$ chromosomes, overexpression of $X$-linked genes due to incomplete inactivation of the second $X$ chromosome, overexpression of miRNAs encoded on $X$ chromosomes, and sex-specific gut microbiota influence the gene expression profile and lead to sex-specific changes in gene expression. These changes in gene expression in turn drive sex-biased autoimmunity. In addition, these factors can influence each other. For example, sex hormones affect the gut microflora and expression of miRNAs and IFN- $\gamma$. In turn, gut microflora can regulate the levels of sex hormones.

Cells of the immune system express estrogen and androgen receptors, and in vitro experiments have tested whether engagement of these receptors affects lymphocyte responses. Estrogen treatment enhances the response to antigen of PBMCs obtained from women (23). Conversely, testosterone inhibits the proliferation and differentiation of lymphocytes, antibody production by B cells, and the cytotoxic activity of NK cells (24-27). Presumably these and similar results are caused by changes in gene expression induced by the hormones, as discussed below.

\section{Sex hormone effects on gene expression and autoimmunity}

Given that the sex hormones bind transcription factors, it is almost certain that these hormones affect autoimmunity via their effects on gene transcription. Many genes have been implicated, some because their transcription is driven directly by hormone nuclear receptors and others because their transcription is controlled indirectly by hormone induced changes in upstream proteins. We will focus first on the genes that are thought to be regulated directly by estrogen or androgen receptors.

\section{IFNs and feedback loops involving sex hormones}

The IFNs are of obvious relevance to this subject because they are well known to be overexpressed in patients with certain autoimmune diseases (SLE in particular; see refs. 28-32) and their absence reduces lupus-like disease in susceptible mice $(33,34)$.

Transcription of IFN1 genes is induced by many pathways, almost all of which involve engagement of pattern recognition receptors (PRRs) such as the TLRs and cytoplasmic sensors of DNA and RNA $(35,36)$. There is not much evidence at present that expression of IFN1 genes is controlled directly by sex hormones. On the other hand, sex hormones do affect expression of some of the PRRs (see below) and in this way might indirectly affect IFN1 levels. Thus, the abundance of IFN1s in lupus patients might be caused by female sex hormone-induced increases in PRR levels, which in turn increase production of IFN1s.

One of the genes that controls expression of IFN1s is IFN regulatory factor 5 (IRF5). IRF5 has also been identified as a significant risk factor for lupus susceptibility $(37,38)$. Expression of IRF5 in mice has been reported to be sex dependent (39). As demonstrated by Shen and colleagues (39), C57BL/6, NZB, Nba2, NZB/NZW F1, and NZM mouse strains express significantly higher levels of Irf5 mRNA in female than in male lymphocytes. Additionally, splenocytes from lupus-prone mice were shown to express higher levels of Irf5 mRNA compared with cells from the C57BL/6 strain, which is not prone to lupus. This group further demonstrated that Irf5 expression can be upregulated in vitro upon estrogen treatment, suggesting a potential mechanism for sex-biased expression of the gene and consequent overproduction of IFN1s (39).

Absence of IFN- $\gamma$ signaling protects NZB/NZW F1 mice against lupus-like disease (40). Expression of the IFNG gene, on the other hand, is regulated directly by estrogens (41-45). This finding suggests a positive feedback loop between the IFNs and estrogens, since activation of IFN1 or IFN- $\gamma$ signaling upregulates the expression of $\mathrm{ER} \alpha$ (46). Estrogen, in turn, promotes IFN- $\gamma$ production by various lymphocytes. In addition, Panchanathan et al. demonstrated synergistic involvement of $\mathrm{ER} \alpha$ and IFN signaling in activating the transcription of both IFN and estrogen-responsive target genes (46).

Once produced, IFNs have many effects on the immune system that contribute to the sex bias of autoimmunity. For example, IFN1s increase class $1 \mathrm{MHC}$ expression on cells, and IFN- $\gamma$ induces class II MHC and changes the nature of the proteasome, thereby affecting the nature and quantity of self-peptides presented to $\mathrm{T}$ cells. Other examples are described below.

\section{Other immune-associated genes affected by sex hormones}

Many genes with products that affect the immune system are controlled by sex hormones. As far as innate immunity is concerned, estrogens induce the expression of intracellular but not surface TLRs in both male and female PBMCs (23). Because intracellular TLRs have been shown to affect the development of autoimmunity (47-51), it is possible that the hormonal effect of the expression of intracellular TLRs contributes to female-biased autoimmunity.

Unc-93 homolog B1 (UNC93B1) is an endoplasmic reticulum (ER) transmembrane protein that is essential for trafficking the TLRs that are expressed intracellularly (TLR3, TLR7, TLR8, TLR9, and probably other TLRs) from the ER to endosomes (52-54). UNC93B1 regulates the activity of these TLRs by mediating localization to the site at which they will be functional. It is thought that this requirement somehow lowers the likelihood that TLRs will respond to host products such as self-nucleic acids that have been taken up as products of dying host cells (54). As far as immune cells are concerned, UNC93B1 is expressed at high levels in B cells, dendritic cells, macrophages, and monocytes (Immunological Genome Project; http://www.immgen.org). 
Several properties of UNC93B1 may be relevant to its possible role in the sex bias of autoimmunity. First, the amount of UNC93B1 protein appears to be limiting, since TLR9 competes with TLR7 for UNC93B1-mediated trafficking to endolysosomes. TLR7 activation drives inflammation in mice in which the preferential binding of UNC93B1 to TLR9 is lost (55). Thus it is expected that increased expression of UNC93B1 would lead to immunological aberrations. Secondly, expression of UNC93B1 is enhanced by estrogen, IFN1s, or IFN- $\gamma$ (56). As mentioned above, moderate amounts of estrogens are known to increase levels of IFNs $(57,58)$ and IFNs are expressed at higher levels in females and autoimmune patients, suggesting the means whereby sex might control expression of UNC93B1. This finding is particularly intriguing because expression of UNC93B1 is markedly elevated in the PBMCs of SLE patients compared with healthy controls (59). Similarly, UNC93B1 levels were appreciably higher in lupus-prone B6.Nba2 female mice when compared with age-matched wild-type controls (56). These observations suggest that UNC93B1 might contribute to female-biased autoimmune responses, perhaps via its ability to increase the concentrations of certain TLRs in endosomes.

Another recent study reports sex differences in the expression of sphingosine-1-phosphate receptor 2 (S1PR2) (60). S1PRs are GPCRs expressed in endothelium and other tissues that regulate cell survival, adherens junction assembly, migration, and barrier integrity (61-63). Several studies have indicated their role in vascular biology (64-66). Using the EAE mouse model for human MS, Cruz-Orengo and colleagues recently suggested a role for S1PR2 in MS (60). SJL mice injected with a myelin oligodendrocyte $G$ peptide develop EAE, and female mice are more susceptible to the disease than males. The investigators showed that S1PR2 expression was increased in the areas of the CNS of the mice that demonstrated damage from EAE in female but not male mice. Moreover, female but not male EAE-induced SJL mice treated with the S1PR2 antagonist JTE-013 exhibited decreased disease severity. In addition, increased levels of S1PR2 were also detected in the CNS of female patients with MS when compared with male patients or healthy controls (60).

In summary, these reports indicate that the expression of a number of genes that play a role in the development of autoimmunity is controlled by sex hormones. However, other gender-specific factors may also contribute to these diseases, as discussed below.

\section{X-linked genes in the sexual dimorphism of autoimmunity}

Apart from the sex hormones themselves, males and females also differ in the numbers of $\mathrm{X}$ or $\mathrm{Y}$ chromosomes each cell contains. Many years ago Mary Lyon suggested that, in order to maintain equivalent expression of $\mathrm{X}$-encoded genes between males and females, one of the $\mathrm{X}$ chromosomes in each female cell should be inactive (67). However, although one of the two X chromosomes in females is almost completely inactive, approximately $15 \%$ of $\mathrm{X}$-linked genes escape inactivation in humans, and a similar phenomenon occurs in mice (68). Escape of these genes is clearly important for female health, as illustrated by Turner syndrome, a chromosomal disorder of females caused by complete or partial loss of an X chromosome (69). Females with the syndrome have several characteristic features and suffer from a number of car- diovascular, renal, and/or neuropsychological disorders (70, 71). Interestingly, patients with Turner syndrome have a higher risk of developing autoimmune diseases than do females in general (72). However, patients with Turner syndrome exhibit a greater risk of autoimmune diseases characterized by male predominance (72), and Turner syndrome rarely overlaps with the profoundly femalebiased disease SLE (73-75).

Symptoms that appear in patients with Klinefelter syndrome also suggest a role for genes on the partially inactivated X chromosome in the immune system. Males with this disorder have two $\mathrm{X}$ and one $\mathrm{Y}$ chromosome. It has been reported that there is a 14-fold increase in the prevalence of SLE in these males compared with the general male population (76). As such, males with the XXY karyotype have a risk of developing SLE that is similar to that of the general female population. In addition, several reports suggest that expression of genes from the partially inactive $\mathrm{X}$ chromosome can play a role in autoimmunity $(77,78)$. Thus, Turner and Klinefelter syndromes indicate that aberrations in the content of $\mathrm{X}$ chromosomes in the host do affect autoimmune disease, although the one-to-one linkage between numbers of $\mathrm{X}$ and $\mathrm{Y}$ chromosomes and particular diseases is still unclear.

Studies establishing a role for X-linked genes lead to the question of whether there are any candidate genes located on the $\mathrm{X}$ chromosome that could potentially play a role in the development of autoimmunity. The $\mathrm{X}$ chromosome encodes many immune-associated genes, including CD4OL, CXCR3, OGT, FOXP3, TLR7, TLR8, IL2RG, BTK, and IL9R (79). Overexpression and/or hypomethylation of $C D 4 O L, C X C R 3$, and $O G T$ have been reported in female but not male patients with $\operatorname{SLE}(77,78)$.

The role of intracellular TLRs in the development of autoimmunity has been extensively studied and is reviewed by others in this issue. In brief, it is well accepted that TLR7 and TLR9 are among the critical players during the development of lupus-like autoimmunity (47). Recent data obtained from different groups suggest that TLR7 is responsible for anti-ribonucleoprotein (antiRNP) and TLR9 for anti-DNA antibody production (47, 48, 80, 81). However, TLR7 and TLR9 play quite different roles in the pathogenesis of murine lupus. TLR9 deficiency leads to the worsening of the disease $(47,48)$, whereas TLR7-deficient animals are partially protected from lupus (47). More recent studies suggest that TLR9 signaling plays a protective role and somehow suppresses the production of TLR7-dependent anti-RNP antibodies $(82,83)$. Several studies have demonstrated that the dosage of $T l r 7$ and Tlr8 plays an important role in the development of both murine and human lupus. For instance, a recent study performed by Umiker and colleagues suggests a role for X-linked Tlr8 dosage in the development of SLE in 564Igi mice (84). Likewise Tlr7 dosage has been demonstrated to play a role in the development of SLE in mice and humans (85-87). Together these data suggest that $T \operatorname{lr} 7$ and Tlr8, due to their localization on $\mathrm{X}$ chromosome, might be overexpressed in females and thus lead to the elevated risk for the development of anti-RNP antibodies and lupus.

Our group has recently described another role for $T \operatorname{lr} 7$ in autoimmunity. In particular, we have identified a subset of B cells (ageassociated B cells [ABCs], discussed in greater detail below), which appear in both aged female wild-type and young autoimmune mice (49). The appearance of these cells is dependent on intact TLR7 sig- 
naling. Moreover, the ablation of ABCs prevents the appearance of autoantibodies in $\mathrm{Mer}^{--}$mice. It is possible that, due to its location on the $\mathrm{X}$ chromosome, $\mathrm{Tl} 7 \mathrm{is}$ overexpressed in females, leading to the accumulation of ABCs in a gender-dependent manner, thereby contributing to female-biased autoimmunity. Further studies will be required to formally test this hypothesis.

Because X chromosome inactivation (XCI) occurs in early embryonic development, one of two $\mathrm{X}$ chromosomes in each cell is inactivated. This is a random and permanent process and, as a result, most females contain a 50:50 mix of cells expressing the $\mathrm{X}$ chromosome of maternal or paternal origin (88). However, some females experience non-random $\mathrm{X}$ chromosome silencing, resulting in $80 \%$ or more cells that are either paternal or maternal in origin, a phenomenon known as skewed XCI. Interestingly, skewed $\mathrm{XCI}$ is associated with autoimmune diseases. For example, $49 \%$ of female scleroderma patients exhibit skewed XCI compared with $2.4 \%$ of healthy controls (89). Significant XCI skewing has also been observed in patients with RA and those with autoimmune thyroiditis $(90,91)$. It is intriguing that severe XCI skewing is also associated with age, and it has been reported that PBMCs of $16 \%$ of females over age of 50 were characterized by skewed XCI (92-94). However, these studies were performed using unseparated PBMCs; therefore, it is still unclear whether different immune cells exhibit similar XCI skewing with age.

\section{X chromosome-encoded microRNAs in sexual dimorphism}

MicroRNAs (miRNAs) may also be governed by sex differences, thereby contributing to susceptibility to autoimmunity. It has been reported that miRNAs are differentially expressed between males and females in both gonadal and non-gonadal tissues (95-97). Several studies indicate numerous dysregulated miRNAs in human and murine lupus, suggesting a role for miRNAs in the development of the disease $(98,99)$. In addition, lupus-associated miRNAs are reported to be differentially expressed in male and female lupusprone NZB/NZW F1 mice (100). Lupus-associated miRNAs regulate the expression of a number of genes that are important for immune responses, including FOXP3, RHOA, FCGR1, and others (101-104). It is not entirely clear what drives differential expression of miRNAs in males and females. The $\mathrm{X}$ chromosome is highly enriched in miRNAs (105): about 7\% (113 miRNAs) of human miRNAs are encoded on the $\mathrm{X}$ chromosome, whereas only two miRNAs have been assigned to the $\mathrm{Y}$ chromosome thus far. Females with SLE are reported to overexpress $18 \mathrm{X}$-linked miRNAs, compared with males with SLE that do not overexpress any miRNAs (78). Although the functions of the majority of X-linked miRNAs remain unknown, some of these miRNAs are reported to play a role in the regulation of immune responses or are associated with autoimmunity (106-111).

Overall the data indicate that the presence of a second X chromosome in females can markedly affect the expression levels of multiple genes and miRNAs, which might be crucial for the development of female-biased autoimmunity.

\section{Gut microbiota: sex differences and influence on autoimmunity}

NOD mice display spontaneous, immune-mediated pancreatic $\beta$ cell destruction, which leads to development of T1D with a strong sex bias (112). The significance of the microbiota for the development of T1D in NOD mice was recently recognized (113). Markle and colleagues demonstrated that germ-free (GF) NOD males and females develop T1D with similar prevalence (114). Moreover, 16S bacterial rRNA sequencing showed that after puberty, male and female NOD mice develop different microbiota profiles. Finally, gavage of female NOD pups with male NOD-derived intestinal microbiota protects the females against development of T1D (114). Another group has reported that hormones mediate sex-based microbiota differences. Castration of male NOD mice reversed the microbiotic differences usually observed between male and female NOD animals (115). These data indicate that sex hormones influence the microbiota in a sex-specific way. In turn, the microbiota causes changes in gene expression that may lead to sex bias in the development of autoimmunity (Figure 1).

An effect of the microbiota has been also reported for the models of ankylosing spondylitis and RA $(116,117)$; however, no effect has been observed in the severity or the prevalence of lupus in GF MRL ${ }^{\mathrm{lpr}}$ mice (118), which indicates that not all autoimmune diseases are affected by microbiota.

\section{Sex-dependent changes in the aging immune system}

Most autoimmune diseases do not develop in childhood but instead affect adults between 40 to 60 years of age. Do male and female immune systems age similarly? Is it possible that some age-related differences occur in sex-dependent fashions, leading to the sex-biased predisposition to autoimmunity?

Two reports indicate that aging affects female and male immune systems differently. A study performed by Yan and colleagues indicated that in both males and females, aging leads to a significant decline in the percentage of naive $\mathrm{CD} 4^{+}$and $\mathrm{CD} 8^{+} \mathrm{T}$ cells and an increase in the percentage of memory T cells, FOXP3 ${ }^{+}$ Tregs, and NK cells (119). However, their data also suggest that the decline in $\mathrm{CD}^{+} \mathrm{T}$ cell frequency and the corresponding increase in the percentage of memory $\mathrm{T}$ cells occurs significantly faster in male than in female subjects (119). Another recent study performed on ethnically Japanese populations of males and females of different ages reported similar age-related changes in immune cell populations. The authors reported age-related declines in $\mathrm{T}$ and $\mathrm{B}$ cell numbers as well as changes in $\mathrm{CD} 4^{+} / \mathrm{CD}^{+} \mathrm{T}$ cell ratios. The rate of decline in $\mathrm{B}$ and $\mathrm{T}$ cell numbers, increases in the $\mathrm{CD} 4^{+} /$ $\mathrm{CD}^{+} \mathrm{T}$ cell ratio, and increases in NK numbers were significantly greater in males versus females (120).

Our group and others have also studied the differences in immune cell populations in aged male and female mice. These data indicate that aged female mice accumulate ABCs (49, 121-123), a subset of B cells defined by the expression of cell surface CD11c and the transcription factor T-bet $(49,123,124)$. We have also reported that $\mathrm{ABCs}$ accumulate in several different mouse models of SLE (MRL ${ }^{\mathrm{lpr}}, \mathrm{NZB} / \mathrm{NZW}, \mathrm{MER}^{-/}$) and coincide with the appearance of autoantibodies $(49,50)$. Moreover, we were able to identify a similar B cell subset in the PBMCs of autoimmune patients. These data lead us to hypothesize that age-associated biological changes in females both contribute to the appearance of ABCs and occur during the onset of autoimmunity. Both processes are sex dependent, which suggests that the same mech- 
anism is involved. We have identified TLR7, IFNGR, and B cell antigen receptor signaling as necessary and sufficient for the upregulation of T-bet expression in B cells $(123,124)$, which eventually causes $\mathrm{B}$ cells to assume the ABC phenotype. We have confirmed that TLR7 is most efficient in driving this process when compared with other TLRs (124). Moreover, the appearance of ABCs is completely dependent on intact TLR7 signaling, since TLR7 deficiency results in the absence of ABCs in both autoimmune and aged wild-type female mice $(49,50)$. This finding is particularly intriguing because the $T l r 7$ gene is encoded on the $\mathrm{X}$ chromosome, offering a potential explanation for the sex bias in the appearance of these cells (123).

It is often noted that some of the female-biased autoimmune diseases are diagnosed in middle-aged individuals, rather than earlier in life when factors such as sex hormones are at peak levels. Some explanations for this paradox have been mentioned above, but it is also worth pointing out that the initial event in autoimmune disease, a breakage in tolerance to self, may actually occur long before the clinical manifestations of the illnesses are manifest. Therefore the crucial problem may indeed occur at a time when estrogens and androgens are at their peak concentrations in the host.

\section{Possible therapeutic interventions}

As detailed above, numerous studies have been performed in an attempt to identify the factors that drive sex bias in autoimmunity. It is important to ask where these reports will lead the field in terms of possible therapeutic interventions. What are the possible outcomes of these studies? The data on the hormonal effects have to be considered when hormonal replacement therapy or treatment with testosterone is used on autoimmune patients. Moreover, the potential immunosuppressive effects of testosterone might make the hormone a useful treatment for patients with autoimmune dis- orders. The data on differential gene expression between genders suggest new potential targets for drug development.

It is likely that the factors reviewed here act simultaneously. Overexpressed X-linked genes and sex hormones probably act together in a synergistic manner, leading to a greater femalebiased predisposition to autoimmunity. At the same time male hormones in combination with a single $\mathrm{X}$ chromosome significantly reduce the risk of autoimmunity. In summary, it is critical to consider all of these factors while developing novel therapeutics for sex-biased autoimmune diseases.

\section{Concluding remarks}

The data reviewed here indicate that immune responses in males and females are differentially regulated by several factors that lead to differences in gene expression profiles (Figure 1). It is important to appreciate both the cause and the outcome of these changes in order to improve our understanding of sex differences in autoimmunity and immune responses in general. More work is required on how sex-specific factors like sex hormones and $\mathrm{X}$ chromosome numbers affect particular populations of immune cells and ultimately lead to the development of autoimmunity, in order to generate novel therapeutic targets for autoimmune disorders that have no cure at the present.

\section{Acknowledgments}

The work was supported in part by US Public Health Service grants AI-18785 and T32 AI074491 (to K. Rubstova).

Address correspondence to: Kira Rubtsova or Philippa Marrack, 1400 Jackson Street, Denver, Colorado 80206, USA. Phone: 303.398.1332; E-mail: rubtsovak@njhealth.org (K. Rubstova), marrackp@njhealth.org (P. Marrack).
1. Oertelt-Prigione S. The influence of sex and gender on the immune response. Autoimmun Rev. 2012;11(6):A479-A485.

2. Fish EN. The X-files in immunity: sex-based differences predispose immune responses. Nat Rev Immunol. 2008;8(9):737-744.

3. Klein SL. The effects of hormones on sex differences in infection: from genes to behavior. Neurosci Biobehav Rev. 2000;24(6):627-638.

4. Chong JW, et al. Marked increase in type 1 diabetes mellitus incidence in children aged 0-14 yr in Victoria, Australia, from 1999 to 2002 . Pediatr Diabetes. 2007;8(2):67-73.

5. Whitacre CC. Sex differences in autoimmune disease. Nat Immunol. 2001;2(9):777-780.

6. Lockshin MD. Sex differences in autoimmune disease. Lupus. 2006;15(11):753-756.

7. Wandell PE, Carlsson AC. Time trends and gender differences in incidence and prevalence of type 1 diabetes in Sweden. Curr Diabetes Rev. 2013;9(4):342-349.

8. Ostman J, et al. Gender differences and temporal variation in the incidence of type 1 diabetes: results of 8012 cases in the nationwide Diabetes Incidence Study in Sweden 1983-2002. J Intern Med. 2008;263(4):386-394.

9. Rubtsov AV, Rubtsova K, Kappler JW, Marrack $P$. Genetic and hormonal factors in female- biased autoimmunity. Autoimmun Rev. 2010;9(7):494-498.

10. Hughes GC, Choubey D. Modulation of autoimmune rheumatic diseases by oestrogen and progesterone. Nat Rev Rheumatol. 2014;10(12):740-751.

11. Hench PS. Effect of jaundice on rheumatoid arthritis. Br Med J. 1938;2(4050):394-398.

12. Confavreux C, Hutchinson M, Hours MM, Cortinovis-Tourniaire P, Moreau T. Rate of pregnancy-related relapse in multiple sclerosis. Pregnancy in Multiple Sclerosis Group. N EnglJ Med.1998;339(5):285-291.

13. Jara LJ, Medina G, Cruz-Dominguez P, Navarro C, Vera-Lastra O, Saavedra MA. Risk factors of systemic lupus erythematosus flares during pregnancy. Immunol Res. 2014;60(2):184-192.

14. Roubinian JR, Talal N, Greenspan JS, Goodman JR, Siiteri PK. Effect of castration and sex hormone treatment on survival, anti-nucleic acid antibodies, and glomerulonephritis in NZB/ NZW F1 mice. J Exp Med. 1978;147(6):1568-1583.

15. Elbourne KB, Keisler D, McMurray RW. Differential effects of estrogen and prolactin on autoimmune disease in the NZB/NZW F1 mouse model of systemic lupus erythematosus. Lupus. 1998;7(6):420-427.

16. Li J, McMurray RW. Effects of estrogen receptor subtype-selective agonists on autoimmune dis- ease in lupus-prone NZB/NZW F1 mouse model. Clin Immunol. 2007;123(2):219-226.

17. Verheul HA, Verveld M, Hoefakker S, Schuurs AH. Effects of ethinylestradiol on the course of spontaneous autoimmune disease in NZB/W and NOD mice. Immunopharmacol Immunotoxicol. 1995;17(1):163-180.

18. Gordon C, et al. Testosterone patches in the management of patients with mild/moderate systemic lupus erythematosus. Rheumatology (Oxford). 2008;47(3):334-338.

19. Sicotte NL, et al. Testosterone treatment in multiple sclerosis: a pilot study. Arch Neurol. 2007;64(5):683-688.

20. Melez KA, Reeves JP, Steinberg AD. Regulation of the expression of autoimmunity in NZB $x$ NZW F1 mice by sex hormones. JImmunopharmacol. 1978;1(1):27-42.

21. Makino S, Kunimoto K, Muraoka Y, Katagiri K. Effect of castration on the appearance of diabetes in NOD mouse. Jikken Dobutsu. 1981;30(2):137-140.

22. Keith RC, et al. Testosterone is protective in the sexually dimorphic development of arthritis and lung disease in SKG mice. Arthritis Rheum. 2013;65(6):1487-1493.

23. Young NA, et al. Estrogen modulation of endosomeassociated toll-like receptor 8: an IFN $\alpha$-independent mechanism of sex-bias in systemic lupus 
erythematosus. Clin Immunol. 2014;151(1):66-77.

24. Sakiani S, Olsen NJ, Kovacs WJ. Gonadal steroids and humoral immunity. Nat Rev Endocrinol. 2013;9(1):56-62.

25. Sthoeger ZM, Chiorazzi N, Lahita RG. Regulation of the immune response by sex hormones. I. In vitro effects of estradiol and testosterone on pokeweed mitogen-induced human B cell differentiation. JImmunol. 1988;141(1):91-98.

26. Calabrese LH, et al. The effects of anabolic steroids and strength training on the human immune response. Med Sci Sports Exerc. 1989;21(4):386-392.

27. Callewaert DM, Moudgil VK, Radcliff G, Waite R. Hormone specific regulation of natural killer cells by cortisol. Direct inactivation of the cytotoxic function of cloned human NK cells without an effect on cellular proliferation. FEBS Lett. 1991;285(1):108-110.

28. Theofilopoulos AN, Baccala R, Beutler B, Kono DH. Type I interferons (alpha/beta) in immunity and autoimmunity. Annu Rev Immunol. 2005;23:307-336

29. Banchereau J, Pascual V. Type I interferon in systemic lupus erythematosus and other autoimmune diseases. Immunity. 2006;25(3):383-392.

30. Crow MK. Type I interferon in the pathogenesis of lupus. J Immunol. 2014;192(12):5459-5468.

31. Hooks JJ, Moutsopoulos HM, Geis SA, Stahl NI, Decker JL, Notkins AL. Immune interferon in the circulation of patients with autoimmune disease. NEngl J Med. 1979;301(1):5-8.

32. Bennett $\mathrm{L}$, et al. Interferon and granulopoiesis signatures in systemic lupus erythematosus blood. JExp Med. 2003;197(6):711-723.

33. Braun D, Geraldes P, Demengeot J. Type I Interferon controls the onset and severity of autoimmune manifestations in lpr mice. J Autoimmun. 2003;20(1):15-25.

34. Santiago-Raber ML, et al. Type-I interferon receptor deficiency reduces lupus-like disease in NZB mice. JExp Med. 2003;197(6):777-788.

35. Trinchieri G. Type I interferon: friend or foe? JExp Med. 2010;207(10):2053-2063.

36. Kawai T, Akira S. The role of pattern-recognition receptors in innate immunity: update on Toll-like receptors. Nat Immunol. 2010;11(5):373-384.

37. Graham RR, et al. A common haplotype of interferon regulatory factor 5 (IRF5) regulates splicing and expression and is associated with increased risk of systemic lupus erythematosus. Nat Genet. 2006;38(5):550-555.

38. Feng D, et al. Genetic variants and disease-associated factors contribute to enhanced interferon regulatory factor 5 expression in blood cells of patients with systemic lupus erythematosus. Arthritis Rheum. 2010;62(2):562-573.

39. Shen H, Panchanathan R, Rajavelu P, Duan X, Gould KA, Choubey D. Gender-dependent expression of murine Irf5 gene: implications for sex bias in autoimmunity. J Mol Cell Biol. 2010;2(5):284-290.

40. Haas C, Ryffel B, Le Hir M. IFN-gamma receptor deletion prevents autoantibody production and glomerulonephritis in lupus-prone (NZB $\times$ NZW) F1 mice. JImmunol. 1998;160(8):3713-3718.

41. Gourdy P, et al. Relevance of sexual dimorphism to regulatory T cells: estradiol promotes IFN- $\gamma$ production by invariant natural killer T cells. Blood. 2005;105(6):2415-2420.

42. Siracusa MC, Overstreet MG, Housseau F, Scott AL, Klein SL. 17 $\beta$-estradiol alters the activity of conventional and IFN-producing killer dendritic cells. JImmunol. 2008;180(3):1423-1431.

43. Nakaya M, Tachibana H, Yamada K. Effect of estrogens on the interferon-gamma producing cell population of mouse splenocytes. Biosci Biotechnol Biochem. 2006;70(1):47-53.

44. Fox HS, Bond BL, Parslow TG. Estrogen regulates the IFN- $\gamma$ promoter. J Immunol. 1991;146(12):4362-4367.

45. Bynote KK, Hackenberg JM, Korach KS, Lubahn DB, Lane PH, Gould KA. Estrogen receptor- $\alpha$ deficiency attenuates autoimmune disease in $(\mathrm{NZB} \times$ NZW)F1 mice. Genes Immun. 2008;9(2):137-152.

46. Panchanathan R, Shen H, Zhang X, Ho SM, Choubey D. Mutually positive regulatory feedback loop between interferons and estrogen receptor-alpha in mice: implications for sex bias in autoimmunity. PLoS One. 2010;5(5):e10868.

47. Christensen SR, Shupe J, Nickerson K, Kashgarian M, Flavell RA, Shlomchik MJ. Toll-like receptor 7 and TLR9 dictate autoantibody specificity and have opposing inflammatory and regulatory roles in a murine model of lupus. Immunity. 2006;25(3):417-428.

48. Christensen SR, Kashgarian M, Alexopoulou L, Flavell RA, Akira S, Shlomchik MJ. Tolllike receptor 9 controls anti-DNA autoantibody production in murine lupus. J Exp Med. 2005;202(2):321-331.

49. Rubtsov AV, et al. Toll-like receptor 7 (TLR7)driven accumulation of a novel CD11c(+) B-cell population is important for the development of autoimmunity. Blood. 2011;118(5):1305-1315.

50. Rubtsov AV, Rubtsova K, Kappler JW, Marrack P. TLR7 drives accumulation of ABCs and autoantibody production in autoimmune-prone mice. Immunol Res. 2013;55(1-3):210-216.

51. Marshak-Rothstein A. Toll-like receptors in systemic autoimmune disease. Nat Rev Immunol. 2006;6(11):823-835.

52. Tabeta K, et al. The Unc93b1 mutation $3 d$ disrupts exogenous antigen presentation and signaling via Toll-like receptors 3, 7 and 9. Nat Immunol. 2006;7(2):156-164.

53. Kim YM, Brinkmann MM, Paquet ME, Ploegh HL. UNC93B1 delivers nucleotide-sensing toll-like receptors to endolysosomes. Nature. 2008;452(7184):234-238.

54. Lee BL, Barton GM. Trafficking of endosomal Toll-like receptors. Trends Cell Biol. 2014;24(6):360-369.

55. Fukui R, et al. Unc93B1 restricts systemic lethal inflammation by orchestrating Toll-like receptor 7 and 9 trafficking. Immunity. 2011;35(1):69-81.

56. Panchanathan R, Liu H, Choubey D. Expression of murine Unc93b1 is up-regulated by interferon and estrogen signaling: implications for sex bias in the development of autoimmunity. Int Immunol. 2013;25(9):521-529.

57. McMurray RW, Hoffman RW, Nelson W, Walker SE. Cytokine mRNA expression in the B/W mouse model of systemic lupus erythematosus - analyses of strain, gender, and age effects. Clin Immunol Immunopathol. 1997;84(3):260-268.
58. Karpuzoglu E, Phillips RA, Gogal RM Jr, Ansar Ahmed S. IFN- $\gamma$-inducing transcription factor, T-bet is upregulated by estrogen in murine splenocytes: role of IL-27 but not IL-12. Mol Immunol. 2007;44(7):1808-1814.

59. Nakano S, Morimoto S, Suzuki S, Watanabe T, Amano H, Takasaki Y. Up-regulation of the endoplasmic reticulum transmembrane protein UNC93B in the B cells of patients with active systemic lupus erythematosus. Rheumatology (Oxford). 2010;49(5):876-881.

60. Cruz-Orengo L, et al. Enhanced sphingosine-1phosphate receptor 2 expression underlies female CNS autoimmunity susceptibility. JClin Invest. 2014;124(6):2571-2584.

61. Garcia JG, et al. Sphingosine 1-phosphate promotes endothelial cell barrier integrity by Edg-dependent cytoskeletal rearrangement. JClin Invest. 2001;108(5):689-701.

62. Lee MJ, et al. Vascular endothelial cell adherens junction assembly and morphogenesis induced by sphingosine-1-phosphate. Cell. 1999;99(3):301-312.

63. Lee MJ, et al. Akt-mediated phosphorylation of the $\mathrm{G}$ protein-coupled receptor EDG-1 is required for endothelial cell chemotaxis. Mol Cell. 2001;8(3):693-704.

64. Zhang G, et al. Critical role of sphingosine-1phosphate receptor 2 (S1PR2) in acute vascular inflammation. Blood. 2013;122(3):443-455.

65. Skoura A, et al. Sphingosine-1-phosphate receptor-2 function in myeloid cells regulates vascular inflammation and atherosclerosis. Arterioscler Thromb Vasc Biol. 2011;31(1):81-85.

66. Kageyama Y, et al. Antagonism of sphingosine 1-phosphate receptor 2 causes a selective reduction of portal vein pressure in bile duct-ligated rodents. Hepatology. 2012;56(4):1427-1438.

67. Lyon MF. Gene action in the X-chromosome of the mouse (Mus musculus L.). Nature. 1961;190:372-373.

68. Carrel L, Willard HF. X-inactivation profile reveals extensive variability in X-linked gene expression in females. Nature. 2005;434(7031):400-404.

69. Stochholm K, Juul S, Juel K, Naeraa RW, Gravholt $\mathrm{CH}$. Prevalence, incidence, diagnostic delay, and mortality in Turner syndrome. JClin Endocrinol Metab. 2006;91(10):3897-3902.

70. Bondy CA, Cheng C. Monosomy for the X chromosome. Chromosome Res. 2009;17(5):649-658.

71. Ranke MB, Saenger P. Turner's syndrome. Lancet. 2001;358(9278):309-314.

72. Jorgensen KT, et al. Autoimmune diseases in women with Turner's syndrome. Arthritis Rheum. 2010;62(3):658-666.

73. Rakfal SM, Deutsch M. Radiotherapy for malignancies associated with lupus: case reports of acute and late reactions. Am J Clin Oncol. 1998;21(1):54-57.

74. Takegami T, et al. [A case of SLE associated with Turner's syndrome of $45, \mathrm{XO} / 46, \mathrm{XXq}+$ mosaicism (author's transl)]. Nihon Naika Gakkai Zasshi. 1980;69(7):861-866.

75. Cooney CM, et al. 46,X,del(X)(q13) Turner's syndrome women with systemic lupus erythematosus in a pedigree multiplex for SLE. Genes Immun. 2009;10(5):478-481. 
76. Scofield RH, et al. Klinefelter's syndrome $(47, \mathrm{XXY})$ in male systemic lupus erythematosus patients: support for the notion of a gene-dose effect from the X chromosome. Arthritis Rheum. 2008;58(8):2511-2517.

77. Lu Q, Wu A, Tesmer L, Ray D, Yousif N, Richardson B. Demethylation of CD4OLG on the inactive $\mathrm{X}$ in $\mathrm{T}$ cells from women with lupus. JImmunol. 2007;179(9):6352-6358.

78. Hewagama A, et al. Overexpression of X-linked genes in T cells from women with lupus. JAutoimmun. 2013;41:60-71.

79. Libert C, Dejager L, Pinheiro I. The X chromosome in immune functions: when a chromosome makes the difference. Nat Rev Immunol. 2010;10(8):594-604.

80. Lartigue A, et al. Role of TLR9 in anti-nucleosome and anti-DNA antibody production in lpr mutation-induced murine lupus. J Immunol. 2006;177(2):1349-1354.

81. Berland R, et al. Toll-like receptor 7-dependent loss of $\mathrm{B}$ cell tolerance in pathogenic autoantibody knockin mice. Immunity. 2006;25(3):429-440.

82. Nickerson KM, et al. TLR9 regulates TLR7- and MyD88-dependent autoantibody production and disease in a murine model of lupus. J Immunol. 2010;184(4):1840-1848.

83. Wu X, Peng SL. Toll-like receptor 9 signaling protects against murine lupus. Arthritis Rheum. 2006;54(1):336-342.

84. Umiker BR, et al. Dosage of X-linked Toll-like receptor 8 determines gender differences in the development of systemic lupus erythematosus. Eur J Immunol. 2014;44(5):1503-1516.

85. Garcia-Ortiz H, Velazquez-Cruz R, EspinosaRosales F, Jimenez-Morales S, Baca V, Orozco L. Association of TLR7 copy number variation with susceptibility to childhood-onset systemic lupus erythematosus in Mexican population. Ann Rheum Dis. 2010;69(10):1861-1865.

86. Shen N, et al. Sex-specific association of X-linked Toll-like receptor 7 (TLR7) with male systemic lupus erythematosus. Proc Natl Acad Sci U S A. 2010;107(36):15838-15843.

87. Subramanian S, et al. A Tlr7 translocation accelerates systemic autoimmunity in murine lupus. Proc Natl Acad Sci U S A. 2006;103(26):9970-9975.

88. Prchal JT, et al. Clonal stability of blood cell lineages indicated by $\mathrm{X}$-chromosomal transcriptional polymorphism. JExp Med.1996;183(2):561-567.

89. Ozbalkan Z, et al. Skewed X chromosome inactivation in blood cells of women with scleroderma. Arthritis Rheum. 2005;52(5):1564-1570.

90. Ozcelik T, et al. Evidence from autoimmune thyroiditis of skewed X-chromosome inactivation in female predisposition to autoimmunity. Eur J Hum Genet. 2006;14(6):791-797.

91. Brix TH, Knudsen GP, Kristiansen M, Kyvik KO, Orstavik KH, Hegedus L. High frequency of skewed X-chromosome inactivation in females with autoimmune thyroid disease: a possible explanation for the female predisposition to thyroid autoimmunity. JClin Endocrinol Metab. 2005;90(11):5949-5953.

92. Chagnon P, Provost S, Belisle C, Bolduc V, Gingras M, Busque L. Age-associated skewing of
$\mathrm{X}$-inactivation ratios of blood cells in normal females: a candidate-gene analysis approach. Exp Hematol. 2005;33(10):1209-1214.

93. Hatakeyama C, Anderson CL, Beever CL, Penaherrera MS, Brown CJ, Robinson WP. The dynamics of X-inactivation skewing as women age. Clin Genet. 2004;66(4):327-332.

94. Sandovici I, Naumova AK, Leppert M, Linares Y, Sapienza C. A longitudinal study of X-inactivation ratio in human females. Hum Genet. 2004;115(5):387-392.

95. Morgan CP, Bale TL. Sex differences in microRNA regulation of gene expression: no smoke, just miRs. Biol Sex Differ. 2012;3(1):22.

96. Cheung L, Gustavsson C, Norstedt G, TolletEgnell P. Sex-different and growth hormoneregulated expression of microRNA in rat liver. BMC Mol Biol. 2009;10:13.

97. Mishima T, et al. MicroRNA (miRNA) cloning analysis reveals sex differences in miRNA expression profiles between adult mouse testis and ovary. Reproduction. 2008;136(6):811-822.

98. Shen N, Liang D, Tang Y, de Vries N, Tak PP. MicroRNAs--novel regulators of systemic lupus erythematosus pathogenesis. Nat Rev Rheumatol. 2012;8(12):701-709.

99. Ceribelli A, Yao B, Dominguez-Gutierrez PR, Nahid MA, Satoh M, Chan EK. MicroRNAs in systemic rheumatic diseases. Arthritis Res Ther. 2011;13(4):229.

100.Dai R, McReynolds S, Leroith T, Heid B, Liang $\mathrm{Z}$, Ahmed SA. Sex differences in the expression of lupus-associated miRNAs in splenocytes from lupus-prone NZB/WF1 mice. Biol Sex Differ. 2013;4(1):19.

101. Xie T, et al. MicroRNA-127 inhibits lung inflammation by targeting Ig G F $\gamma \gamma$ receptor I. J Immunol. 2012;188(5):2437-2444.

102. Rouas R, et al. Human natural Treg microRNA signature: role of microRNA-31 and microRNA-21 in FOXP3 expression. Eur J Immunol. 2009;39(6):1608-1618.

103. Fan W, et al. Identification of microRNA-31 as a novel regulator contributing to impaired interleukin-2 production in $\mathrm{T}$ cells from patients with systemic lupus erythematosus. Arthritis Rheum. 2012;64(11):3715-3725.

104.Dai R, et al. Identification of a common lupus disease-associated microRNA expression pattern in three different murine models of lupus. PLoS One. 2010;5(12):e14302.

105.Pinheiro I, Dejager L, Libert C. X-chromosomelocated microRNAs in immunity: might they explain male/female differences? The $\mathrm{X}$ chromosome-genomic context may affect $\mathrm{X}$-located miRNAs and downstream signaling, thereby contributing to the enhanced immune response of females. Bioessays. 2011;33(11):791-802.

106. Fazi F, et al. A minicircuitry comprised of microRNA-223 and transcription factors NFI-A and $\mathrm{C} / \mathrm{EBP} \alpha$ regulates human granulopoiesis. Cell. 2005;123(5):819-831.

107. Fulci V, et al. miR-223 is overexpressed in T-lymphocytes of patients affected by rheumatoid arthritis. Hum Immunol. 2010;71(2):206-211. 108. Wang H, Peng W, Ouyang X, Li W, Dai Y. Cir- culating microRNAs as candidate biomarkers in patients with systemic lupus erythematosus. Transl Res. 2012;160(3):198-206.

109. Pandis I, et al. Identification of microRNA-221/222 and microRNA-323-3p association with rheumatoid arthritis via predictions using the human tumour necrosis factor transgenic mouse model. Ann Rheum Dis. 2012;71(10):1716-1723.

110. Lu C, et al. miR-221 and miR-155 regulate human dendritic cell development, apoptosis, and IL-12 production through targeting of p27kip1, KPC1, and SOCS-1. Blood.2011;117(16):4293-4303.

111. Grigoryev YA, et al. MicroRNA regulation of molecular networks mapped by global microRNA, mRNA, and protein expression in activated Tlymphocytes. JImmunol. 2011;187(5):2233-2243.

112. Pozzilli P, Signore A, Williams AJ, Beales PE. NOD mouse colonies around the world - recent facts and figures. Immunol Today. 1993;14(5):193-196.

113. Wen L, et al. Innate immunity and intestinal microbiota in the development of Type 1 diabetes. Nature. 2008;455(7216):1109-1113.

114. Markle JG, et al. Sex differences in the gut microbiome drive hormone-dependent regulation of autoimmunity. Science. 2013;339(6123):1084-1088.

115. Yurkovetskiy L, et al. Gender bias in autoimmunity is influenced by microbiota. Immunity. 2013;39(2):400-412.

116. Rehakova Z, et al. Germ-free mice do not develop ankylosing enthesopathy, a spontaneous joint disease. Hum Immunol. 2000;61(6):555-558.

117. Abdollahi-Roodsaz S, et al. Stimulation of TLR2 and TLR4 differentially skews the balance of $\mathrm{T}$ cells in a mouse model of arthritis. JClin Invest. 2008;118(1):205-216.

118. Maldonado MA, et al. The role of environmental antigens in the spontaneous development of autoimmunity in MRL-lpr mice. J Immunol. 1999;162(11):6322-6330.

119. Yan J, et al. The effect of ageing on human lymphocyte subsets: comparison of males and females. Immun Ageing. 2010;7:4.

120. Hirokawa K, Utsuyama M, Hayashi Y, Kitagawa M, Makinodan T, Fulop T. Slower immune system aging in women versus men in the Japanese population. Immun Ageing. 2013;10(1):19.

121. Hao Y, O'Neill P, Naradikian MS, Scholz JL, Cancro MP. A B-cell subset uniquely responsive to innate stimuli accumulates in aged mice. Blood. 2011;118(5):1294-1304

122. Rubtsova K, Marrack P, Rubtsov AV. Age-associated B cells: are they the key to understanding why autoimmune diseases are more prevalent in women? Expert Rev Clin Immunol. 2012;8(1):5-7.

123. Rubtsova K, Marrack P, Rubtsov AV. TLR7, IFN $\gamma$, and T-bet: Their roles in the development of ABCs in female-biased autoimmunity [published online ahead of print December 13, 2014]. Cell Immunol. doi:10.1016/j.cellimm.2014.12.002.

124. Rubtsova K, Rubtsov AV, van Dyk LF, Kappler JW, Marrack P. T-box transcription factor T-bet, a key player in a unique type of B-cell activation essential for effective viral clearance. Proc Natl Acad Sci U S A. 2013;110(34):E3216-E3224. 